Note

\title{
Establishment and Evaluation of Event-Specific Quantitative PCR Method for Genetically Modified Soybean MON89788
}

(Received March 23, 2010)

\author{
Reona Takabatake ${ }^{1}$, Mari Onishi ${ }^{2}$, Tomohiro Koiwa ${ }^{3}$, Satoshi Futo ${ }^{2}$, \\ Yasutaka Minegishi ${ }^{4}$, Hiroshi AkiYama ${ }^{5}$, Reiko Teshima ${ }^{5}$, \\ Satoshi Furui ${ }^{1}$ and Kazumi Kitta ${ }^{1, *}$ \\ ${ }^{1}$ Analytical Science Division, National Food Research Institute, National Agriculture and \\ Food Research Organization: 2-1-12 Kannondai, Tsukuba, Ibaraki 305-8642, Japan; \\ ${ }^{2}$ Fasmac Co., Ltd.: 5-1-3 Midorigaoka, Atsugi, Kanagawa 243-0041, Japan; \\ ${ }^{3}$ Food and Agricultural Materials Inspection Center: 2-1 Shintoshin, \\ Chuo-ku, Saitama 330-9731, Japan; \\ ${ }^{4}$ Nippon Gene Co., Ltd.: 1-8-7 Toiyamachi, Toyama 930-0834, Japan; \\ ${ }^{5}$ National Institute of Health Sciences: 1-81-1 Kamiyoga, \\ Setagaya-ku, Tokyo 158-8501, Japan; \\ ${ }^{*}$ Corresponding author
}

\begin{abstract}
A novel real-time PCR-based analytical method was established for the event-specific quantification of a GM soybean event MON89788. The conversion factor $\left(C_{\mathrm{f}}\right)$ which is required to calculate the GMO amount was experimentally determined. The quantitative method was evaluated by a single-laboratory analysis and a blind test in a multi-laboratory trial. The limit of quantitation for the method was estimated to be $0.1 \%$ or lower. The trueness and precision were evaluated as the bias and reproducibility of the relative standard deviation $\left(\mathrm{RSD}_{\mathrm{R}}\right)$, and the determined bias and $\mathrm{RSD}_{\mathrm{R}}$ values for the method were both less than $20 \%$. These results suggest that the established method would be suitable for practical detection and quantification of MON 89788.
\end{abstract}

Key words: MON89788; event-specific; genetically modified (GM); real-time PCR; soybean

\section{Introduction}

Glyphosate is a well-known herbicide which is active against a broad spectrum of plant species, and glyphosate tolerance has been genetically engineered into various crops such as canola, cotton, maize, and soybean ${ }^{1)}$. A glyphosate-tolerant soybean (GTS), event 40-3-2 [Roundup Ready Soy (RRS)], is one of the most successful genetically modified (GM) crops and has become dominant in several countries where its planting is permitted ${ }^{1}$. Indeed, the GM soybean-planted area made up more than half of the total GM-planted area in $2007^{2}$. Based on experience of using RRS and the benefits gained from its use, a second-generation GTS, MON89788, has been developed, and is now being commercially cultivated. MON89788 integrates the $c p 4$ epsps gene, the chimeric promoter consisting of the enhancer sequence of the Figwort Mosaic Virus (FMV) promoter bound to the Tsf1 promoter derived from Arabidopsis thaliana, and the terminator from pea ribulose 1,5-bisphosphate carboxylase (TRbcS) at a single locus in the soybean genome ${ }^{3)}$. It is reported that MON89788 enables the same flexible, dependable weed control as the original RRS, but with higher yield potential resulting from advanced gene mapping and insertion technology*1, and the relative crop yield of MON 89788 is increased from 7 to $11 \%$ over RRS*2. Therefore, MON89788 is expected to become widely used.

There are requirements for labeling GM products in crops or derived products in the European Union (EU), Korea, Japan, Australia, and many countries. The threshold for the unintentional commingling level of GM crops was set at 5\% in Japan ${ }^{4) * 3}$. To date, real-time PCR-based detection and quantitative methods for several GM maize events and RRS have already been developed and validated by our international interlaboratory collaborative study ${ }^{5), 6)}$, and adopted as

*1 Monsanto Co., news release http: / / monsanto. mediaroom. com $/$ index. php? $\mathrm{s}=43 \&$ item $=687$

*2 Nougyou To Kankyou No. 90, National Institute for Agro-Enviromental Sciences http: / / www. niaes. affrc. go. jp / magazine / 090 / mgzn 09008.html

*3 Notification No. 517 (Mar. 31, 2000); Ministry of Agriculture, Forestry and Fisheries of Japan (2000) 
Japanese standard analytical methods $* 4, * 5$. However, in Japan there is no standard method for detecting MON89788. An analytical method to detect MON89788 is needed for practical monitoring because MON89788 cannot be detected even by using target sequences from Cauliflower Mosaic Virus 35S promoter (P35S) and the nopaline synthase terminator regions which are commonly used in many GM crops.

In this report, we describe a new quantitative method for detecting MON89788 with an event-specific PCR strategy, and an evaluation of the method.

\section{Materials and Methods}

\section{Plant materials}

GM soybean and maize seeds, MON89788, RRS, MON 810, MON863, MON88017, and NK603 were kindly provided by the Monsanto Company (St. Louis, MO, USA), A2704-12 by its developer, Bt11, Event176, GA21, and MIR604 by Syngenta Seeds AG (Basel, Switzerland), and TC1507 and DAS59122 by Pioneer Hi-Bred International (Johnston, IA, USA). T25 was directly imported from the USA. Dry soybean seeds which were harvested in the USA in 2004, were purchased from Ryokokushoji Co., Ltd. (Hiroshima, Japan) and used as a non-GM soy sample.

\section{Oligonucleotide primers and probes}

For specific detection of MON89788, the primers (MON89788-F; 5' -TCCCGCTCTAGCGCTTCAAT-3' and MON89788-R; 5'-TCGAGCAGGACCTGCAGAA-3') and fluorescent dye-labeled probe (MON89788-P; 5'-CTGAAGGCGGGAAACGACAATCTG-3') were used for real-time PCR. The sequences of these primers and probe were taken from the report of the European Commission's Joint Research Center (JRC, IRMM, Retieseweg, Geel, Belgium)*6. The soy lectin1 $(\text { Le1 })^{7)}$ was used as a soybean-specific endogenous reference DNA for qualitative and quantitative analyses. For specific detection of Le1, the primers (Le1n02-5'; $5^{\prime}$ GCCCTCTACTCCACCCCCA-3' and Le1n02-3'; 5'GCCCATCTGCAAGCCTTTTT-3') and fluorescent dyelabeled probe (Le1-Taq; 5'-AGCTTCGCCGCTTCCTTCAACTTCAC-3') were used. The maize starch synthase $I I b$ (SSIIb) gene was used as a maize-specific endogenous DNA for qualitative analysis. For specific detection of

*4 Notification No. 110 (Mar. 27, 2001); Department of Food Safety, Ministry of Health, Labour and Welfare of Japan (2001)

*5 Japanese Agricultural Standard (JAS) analytical test handbook: genetically modified food quality, labeling analysis manual for individual products (2002)

The Food and Agricultural Materials Inspection Center, Japan.

http: / / www. famic. go. jp / technical_ information / jashandbook/index.html

*6 Event-specific method for the quantification of soybean line MON89788 using real-time PCR. http://gmo-crl. jrc. ec. europa. eu / summaries / MON 89788 _ validated _ Method.pdf
SSIIb, the primers (SSIIb3-5'; 5'-CCAATCCTTTGACATCTGCTCC-3' and SSIIb3-3'; 5'-GATCAGCTTTGGGTCCGGA-3 $\left.{ }^{\prime}\right)^{6}$ were used. The oligonucleotide primers and TaqMan ${ }^{\circledR}$ probes were synthesized by Fasmac Co., Ltd. (Kanagawa, Japan) and Life Technologies (Carlsbad, CA, USA), respectively. The oligonucleotide probes were labeled with 6-carboxyfluorescein (FAM) at the $5^{\prime}$ ends and 6-carboxytetramethylrhodamine (TAMRA) at the $3^{\prime}$ ends.

\section{Preparation of calibrant plasmid}

The specific sequence fragments from MON89788, endogenous soybean Le1 gene ${ }^{8)}$, and $\mathrm{P}_{35 \mathrm{~S}^{8)}}$ were synthesized as a single oligonucleotide in tandem and inserted into the pUC19 vector. The constructed plasmid, designated as pSCS, was purified and diluted with $5 \mathrm{ng}$ / $\mu \mathrm{L}$ ColE1 DNA (Nippon Gene, Tokyo, Japan) solution to $20,125,1500,20,000$, and 250,000 copies per $2.5 \mu \mathrm{L}$, based on the copy number of the Le1 segment.

\section{Preparation of test samples}

For evaluation of the quantitative method, DNA solutions consisting of different concentrations of extracted MON89788 DNA in non-GM soybean DNA were used. MON89788 and non-GM soybean genomic DNA was extracted from ground seeds using GM Quicker (Nippon Gene) according to the manufacturer's manual. The concentration and quality of the extracted DNA solutions were evaluated by measuring the ultraviolet (UV) absorbance with a spectrophotometer, DU800 (Beckman Coulter, Fullerton, CA, USA) as described previously ${ }^{6)}$. Both MON89788 and the non-GM soybean genomic DNA solutions were adjusted to a concentration of $20 \mathrm{ng} / \mu \mathrm{L}$, and then mixed. Samples containing a mixture of the genomic DNA solutions at concentrations of MON89788 of $0,0.1,0.5,5.0$, and $10.0 \%$ in volume ratio were prepared. The mixed samples of each concentration were divided into 24 sample tubes, and six tubes were then randomly selected for a single laboratory evaluation and multi-laboratory trial, respectively.

\section{Qualitative PCR}

Qualitative PCR using a thermal cycler, GeneAmp PCR system 9700 (Life Technologies) and agarose gel electrophoresis were performed as described by Kuribara et al. ${ }^{8}$.

\section{Quantitative PCR}

TaqMan real-time PCR assays were carried out using an ABI PRISM 7900HT (Life Technologies), in $25 \mu \mathrm{L}$ final volume, with $50 \mathrm{ng}$ of sample DNA, $12.5 \mu \mathrm{L}$ Universal Master Mix (Life Technologies), $0.5 \mu \mathrm{M}$ primer pairs and $0.2 \mu \mathrm{M}$ probe, according to the following stepcycle program: $2 \mathrm{~min}$ at $50^{\circ} \mathrm{C}, 10 \mathrm{~min}$ at $95^{\circ} \mathrm{C}, 45$ cycles, $30 \mathrm{sec}$ at $95^{\circ} \mathrm{C}$, and $1 \mathrm{~min}$ at $59^{\circ} \mathrm{C}$. In the reaction plate, each sample was quantified in triplicate. 


\section{Multi-laboratory trial}

A multi-laboratory trial was performed at 3 laboratories and consisted of 2 separate stages, a stage to measure the $C_{\mathrm{f}}$ value and a blind test. All experimental protocols were provided by the National Food Research Institute (NFRI). Quantitative real-time PCR was performed with primers, probes, Universal Master Mix and DNA solutions supplied by NFRI.

The $C_{\mathrm{f}}$ value is experimentally determined as the ratio of the copy number of recombinant DNA (r-DNA) to the taxon-specific sequence in the GM plant genome. To calculate the $C_{\mathrm{f}}$ value for MON89788, the genomic DNA was extracted from genuine GM seeds, and the copy numbers of r-DNA and taxon-specific sequences were measured. The measurement was done twice at 3 laboratories, and the $C_{\mathrm{f}}$ value was determined as the mean of the 6 values obtained.

A blind test was carried out as described previously ${ }^{6)}$. Blind samples which were designed as blind duplicates of the 5 different concentrations of MON89788 DNA, consisting of $0,0.1,0.5,5.0$, and $10.0 \%$, were sent to the participants.

All participants were requested to submit their data from the real-time PCR analyses.

\section{Results and Discussion}

\section{Specificity of the PCR system for MON89788}

For specific detection of MON89788, an event-specific segment was amplified at the junction site between the native soybean genomic DNA and r-DNA (Fig. 1). The specificity of the primer set was confirmed by a qualitative PCR method. No unexpected PCR products were detected using genomic DNA solutions from non-GM soybean, GM soybean RRS and A2704-12, non-GM maize, or 11 lines of GM maize, including glyphosatetolerant events such as GA21 and NK603 (Fig. 2).

\section{Determination of $C_{\mathrm{f}}$ value for MON89788}

To determine the experimental $C_{\mathrm{f}}$ value for MON 89788, the copy numbers of Le1 and MON89788 in the extracted DNA from the MON89788 seeds were measured.

In Japan, one of the features of standard quantitative methods is utilization of standard plasmid DNA containing both r-DNA and endogenous sequences as reference molecules and calibrators. To harmonize with the Japanese standard method, we developed one novel standard plasmid. The pSCS plasmid includes the specific sequences of MON89788 (Fig. 3), the Le1 segment, and the P35S segment. The measurements were repeated twice, and the $C_{\mathrm{f}}$ value was determined as the mean from three laboratories. The defined $C_{\mathrm{f}}$ value for MON 89788 was 1.21 (Table 1), which was used in the following quantifications.

\section{Single laboratory evaluation of the PCR quantification}

We performed single-laboratory and multi-laboratory evaluations of the proposed quantitative method using samples prepared by mixing extracted DNAs of GM and

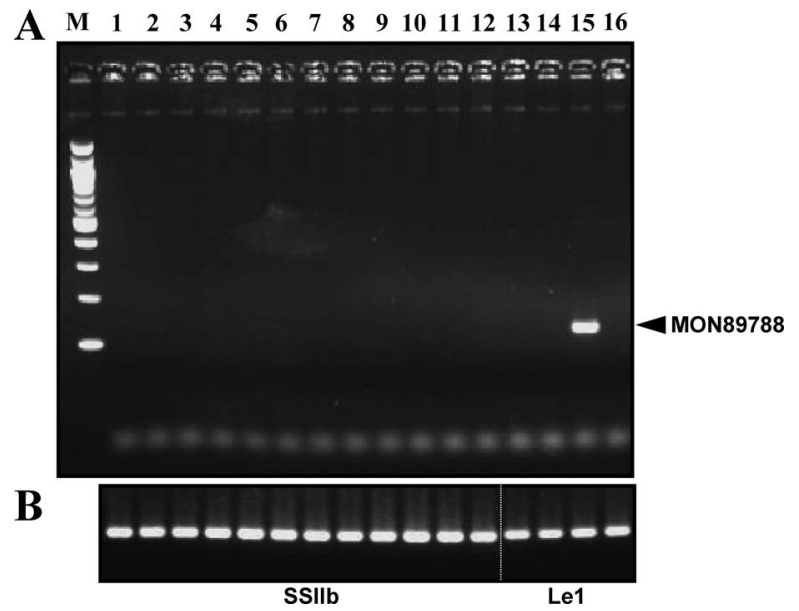

Fig. 2. Specificity test of the designed primer. Agarose gel (3.0\%) electrophoretogram of the amplified PCR products corresponding to the $139 \mathrm{bp}$ of MON89788 (A), $114 \mathrm{bp}$ of SSIIb and $118 \mathrm{bp}$ of Le1 in lanes $1-12$ and $13-16$ of (B), respectively. Lanes 1-11, GM maize events, NK603, Event176, T25, GA21, MON810, TC1507, Bt11, MIR604, MON88017, DAS59122, and MON863, respectively; 12 and 13, non-GM maize and non-GM soy, respectively; 14-16, GM soybean events, RRS, MON89788, and A2704-12, respectively. M, molecular weight marker.
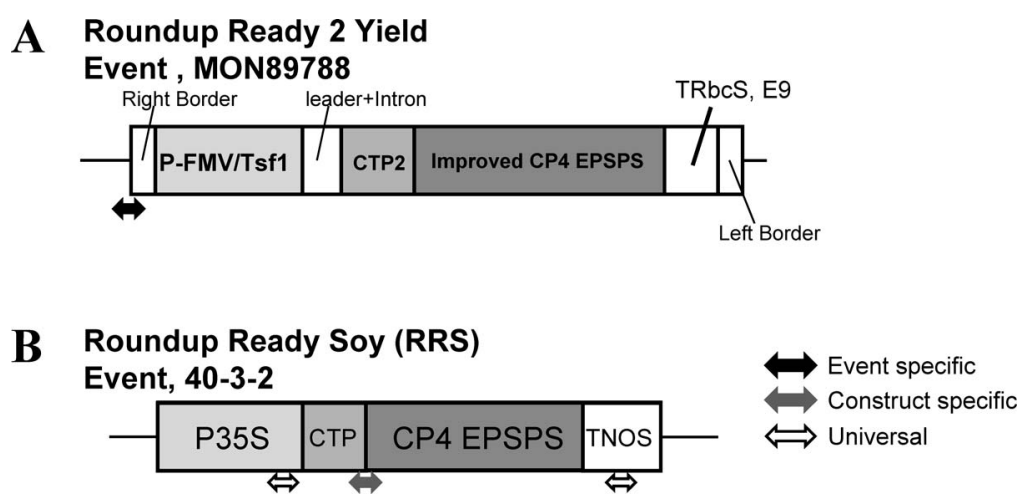

Fig. 1. Schematic diagrams of the recombinant DNA of MON89788 and RRS. Arrows indicate the target positions in MON89788 (A) and RRS (B). Event-specific target sequence of MON89788 was designed for the $5^{\prime}$-flanking region between the exogenous insert and host soybean DNA. 


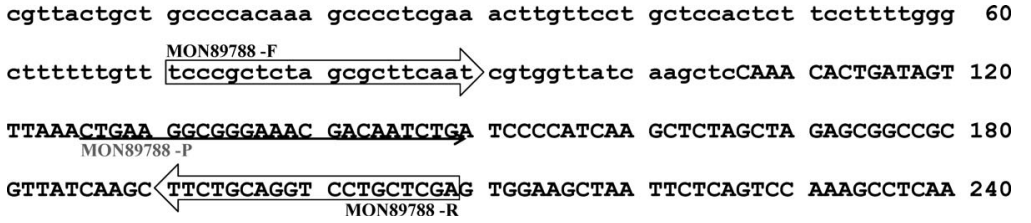

Fig. 3. Sequence between the $5^{\prime}$ exogenous insertion and the soybean genome of MON89788. Lowercase letters represent the flanking soybean genomic sequence, and capital letters show the $5^{\prime}$ transgenic insert containing the right border and partial FMV promoter sequences. The primers and probe for MON89788 are boxed and underlined, respectively.

non-GM DNA solutions. We decided that using DNA solution-based mixing samples would be easy and snitable for evaluation of the method.

In the single-laboratory evaluation, the GMO amount (\%) of each sample was analyzed in six repetitions, and statistical analyses were carried out. The mean, bias (mean value, \%), and relative standard deviation (RSD) at each mixing level were calculated (Table 2). The largest value of determined bias from the true value was 13.3 , and the highest value of the RSD was $14.4 \%$ for the $0.1 \%$ mixing sample.

\section{Blind test}

We next evaluated the proposed quantitative method with a blind test involving 3 laboratories. All participants received primers, probes, and the test samples consisting of 5 different concentrations of MON89788, and the measurement was performed twice. The true-

Table 1. Experimental conversion factor for MON89788

\begin{tabular}{ccc}
\hline \hline & $7900 \mathrm{HT}$ & \\
\hline Mean & $\mathrm{SD}$ & $\mathrm{RSD}$ \\
\hline 1.21 & 0.0866 & 7.14 \\
\hline
\end{tabular}

SD; Standard deviation

RSD; Relative standard deviation ness and precision were determined as previously described $^{5), 6)}$. The bias, repeatability $\mathrm{RSD}\left(\mathrm{RSD}_{\mathrm{r}}\right)$, and reproducibility $\mathrm{RSD}\left(\mathrm{RSD}_{\mathrm{R}}\right)$ of the blind samples were measured (Table 3). The determined bias, $\mathrm{RSD}_{\mathrm{r}}$, and $\mathrm{RSD}_{\mathrm{R}}$ ranged from -11.7 to $5.00 \%$, from 2.70 to $19.4 \%$, and from 5.92 to $19.4 \%$, respectively. In Europe, the acceptance criteria for full validation of an analytical method are defined by the European Network of GMO Laboratories (ENGL)*7. All of the obtained bias values were below the limit of the trueness acceptable level in

Table 2. Single-laboratory evaluation

\begin{tabular}{|c|c|c|c|c|c|}
\hline \multirow{2}{*}{$\begin{array}{c}\% \\
(\mathrm{v} / \mathrm{v})\end{array}$} & \multirow[b]{2}{*}{$n$} & Means & Bias & \multirow{2}{*}{$\begin{array}{c}\mathrm{RSD}^{a} \\
\%\end{array}$} & $\begin{array}{c}\text { Detection } \\
\text { limit }\end{array}$ \\
\hline & & $\begin{array}{c}\text { GMO } \\
\text { amount, } \\
\%\end{array}$ & $\begin{array}{c}\text { True } \\
\text { value, } \\
\%\end{array}$ & & $\begin{array}{c}\text { Below } 20 \\
\text { copies }^{b}\end{array}$ \\
\hline 0.00 & 6 & 0.00 & 0.00 & nd & $6 / 6$ \\
\hline 0.10 & 6 & 0.113 & 13.3 & 14.4 & $0 / 6$ \\
\hline 0.50 & 6 & 0.532 & 6.34 & 4.96 & $0 / 6$ \\
\hline 5.0 & 6 & 5.00 & -0.06 & 9.03 & $0 / 6$ \\
\hline 10.0 & 6 & 9.37 & -6.35 & 8.40 & $0 / 6$ \\
\hline
\end{tabular}

${ }^{a}$ RSD: Relative standard deviation

${ }^{b}$ Below 20 copies are expressed as the ratio of the number of retained data below 20 copies/the total number of retained data.

Table 3. Trueness and precision

\begin{tabular}{|c|c|c|c|c|c|c|}
\hline \multirow[b]{3}{*}{$\begin{array}{c}\% \\
(\mathrm{v} / \mathrm{v})\end{array}$} & \multirow[b]{3}{*}{$\begin{array}{l}\text { Retained } \\
\text { labs }\end{array}$} & \multicolumn{2}{|c|}{ Trueness } & \multicolumn{2}{|c|}{ Precision } & \multirow{2}{*}{$\begin{array}{l}\text { Detection } \\
\text { limit }\end{array}$} \\
\hline & & Means & Bias & & & \\
\hline & & $\begin{array}{c}\text { GMO } \\
\text { amount, } \\
\%\end{array}$ & $\begin{array}{c}\text { True } \\
\text { value, } \\
\%\end{array}$ & $\begin{array}{l}\mathrm{RSD}_{\mathrm{r}}{ }^{a}, \\
\%\end{array}$ & $\begin{array}{c}\mathrm{RSD}_{\mathrm{R}}{ }^{b} \\
\%\end{array}$ & $\begin{array}{c}\text { Below } 20 \\
\text { copies }^{c}\end{array}$ \\
\hline 0.00 & 3 & 0.00 & 0.00 & nd & nd & $6 / 6$ \\
\hline 0.10 & 3 & 0.105 & 5.00 & 19.4 & 19.4 & $0 / 6$ \\
\hline 0.50 & 3 & 0.442 & -11.7 & 16.8 & 16.8 & $0 / 6$ \\
\hline 5.0 & 3 & 4.85 & -3.00 & 2.70 & 5.92 & $0 / 6$ \\
\hline 10.0 & 3 & 9.18 & -8.20 & 5.19 & 6.32 & $0 / 6$ \\
\hline
\end{tabular}

${ }^{a} \mathrm{RSD}_{\mathrm{r}}$ : Repeatability relative standard deviation

${ }^{b} \mathrm{RSD}_{\mathrm{R}}$ : Reproducibility relative standard deviation

${ }^{c}$ Below 20 copies are expressed as the ratio of the number of retained data below 20 copies/the total number of retained data.

When $\mathrm{RSD}_{\mathrm{r}}>\mathrm{RSD}_{\mathrm{R}}, \mathrm{RSD}_{\mathrm{R}}$ was considered to have the same value of $\mathrm{RSD}_{\mathrm{r}}{ }^{9}$.

*7 Definition of Minimum Performance Requirements for Analytical Methods of GMO Testing, European Network of GMO Laboratories (ENGL)

http://gmo-crl.jrc.ec.europa.eu/doc/Min_Perf_Requirements_Analytical_methods.pdf 
ENGL (The bias value should be within $\pm 25 \%$ ). The obtained $\mathrm{RSD}_{\mathrm{r}}$ and $\mathrm{RSD}_{\mathrm{R}}$ here were similar to, or within a narrower range than, those in previously reported GMO events ${ }^{5), 6)}$. ISO $24276^{* 8}$ specifies that the $\mathrm{RSD}_{\mathrm{R}}$ is $25 \%$ or less at the limit of quantitation (LOQ) in GMO analysis, and all the obtained $\mathrm{RSD}_{\mathrm{R}}$ values were within the criterion, even for $0.10 \%$ samples. The data below 20 copies were extrapolated based on the standard curve in our method because there was no calibrant below 20 copies. In Table 3, all the measured copy numbers of $0.10 \%$ samples were over 20 copies. Therefore, it was estimated that the LOQ for MON89788 is $0.10 \%$ in this method.

In conclusion, the newly developed method is applicable for the detection and quantification of MON89788 to monitor the validity of the labeling. Several GM soybean events such as RRS, MON89788, and gluphosinate-tolerant soybeans have been approved in Japan. The number of approved GM soybean events is expected to steadily increase. Specific analytical methods for novel GM events or a screening method for GM soybean events will be required.

\section{Acknowledgements}

We would like to thank the following collaborators for their participation in this study:

Fasmac Co., Ltd., Kanagawa, Japan

Food and Agricultural Materials Inspection Center, Saitama, Japan

National Institute of Health Sciences, Tokyo, Japan

We would also like to thank Shigehiro Naito (National Food Research Institute, Ibaraki, Japan) for his excellent suggestions regarding the statistical aspects of this study. This work was supported by the Ministry of Agriculture, Forestry, and Fisheries of Japan Research Project, "Assurance of Safe Use of Genetically Modified Organisms", and by a Grant from the Ministry of Health, Labor and Welfare of Japan.

\section{References}

1) Cerdeira, A. L., Duke S. O. The current status and environmental impacts of glyphosate-resistant crops: A review, 35, 1633-1658 (2006).

2) James, C. Executive summary of global status of commercialized biotech/GM crops. ISAAA Briefs 2007, No. 37.

3) Malven, M., Rinehart, J., Taylor, N., Dickinson, E. Soybean event MON89788 and methods for detection thereof. Patent No. WO 2006/130436 A2,2006.

4) Hino, A. Safety assessment and public concerns for genetically modified food products: The Japanese experience. Toxicol. Pathol., 30, 126-128 (2002).

5) Shindo, Y., Kuribara, H., Matsuoka, T., Futo, S., Sawada, C., Shono, J., Akiyama, H, Goda, Y., Toyoda, M., Hino, A. Validation of real-time PCR analyses for line-specific quantitation of genetically modified maize and soybean using new reference molecules. J. AOAC Int., 85, 11191126 (2002).

6) Kodama, T., Kuribara, H., Minegishi, Y., Futo, S., Watai, M., Sawada, C., Watanabe, T., Akiyama, H., Maitani, T., Teshima, R., Furui, S., Hino, A., Kitta, K. Evaluation of modified PCR quantitation of genetically modified maize and soybean using reference molecules: interlaboratory study. J. AOAC Int., 92, 223-233 (2009).

7) Vodkin L. O., Rhodes P. R., Goldberg, R. B. cA lectin gene insertion has the structural features of a transposable element. Cell, 34, 1023-1031 (1983).

8) Kuribara, H., Shindo, Y., Matsuoka, T., Takubo, K., Futo, S., Aoki, N., Hirao, T., Akiyama, H., Goda, Y., Toyoda, M., Hino, A. Novel reference molecules for quantification of genetically modified maize and soybean. J. AOAC Int., 85, 1077-1089 (2002).

9) Horwitz, W. Protocol for the design, conduct and interpretation of method-performance Studies. Pure \& Appl. Chem., 67, 331-343 (1995).

*8 International Standard 24276, Foodstuffs-Nucleic acid based of analysis for the detection of genetically modified organisms and derived products-General requirements and definitions 